ante un problema de clinica Obstétrica. Es una obligación de los especialistas llevar esto a conocimiento del público y éste tiene el derecho de repudiar a quienes no saben justipreciarse o no quieren hacerlo, y pretenden medrar a la sombra del alto porcentaje de normalidad con Que se cumple el parto, amparados por la falta de legislación n to to. cante al ejercicio de las especializaciones $y$, lo decimos con énfasis, a la falta de legislación sabre responsabilidad profesional. QUIEN NO SABE VALORARSE PECA POR IG. NORANCIA O POR FALTA DE HONRADEZ, to ha dicho también Stoeckel.

\title{
Consideraciones sobre la lrombosis puerperal y la embolia pulmonar
}

Por Alberto Cárdenas Escuvar

Entre las complicaciones del puerperio, una de las más molestas, rebeldes $y$ peligrosas es la llamada generaimente "Flebitis puerperal". Aungue se la ha considerado por rrucho tiempo como una iorma de It infección. actualmente se acepta su division en dos procesos distintos. a saber:

$1^{0}$ us simple ficbo-trombosis, tambien llamada "irombosis blanda". que corresponde al "tipo no inflanatorio" de algunos atutures, $y$ consisto en la formación de cougulo dentro de la vena. La patogenia no se conoce del todo, pero es probable que dos factores iniciales importan. tes sean la estasis venosa y lesiones pre-existentes en las veras. Se sabe gue cespues de la expulsion de la piranta, la sangre se coagula en los senos venosos del are placontaria acluyendo los vasos abiertos; normimente el cotrulo se extente has. ta cierta profundiad en el espesor de la pared uterina. Pero puede ocurrir que también se forme coágulo más alla de este límite, resultando entonces afectridos otros troncos venosos como las iliacas internas, iliacas primitivas, iliacas externas y femorales. La trombosis femoral está descrita en muchos textos como tipo de esta complicacion puerperal. A veces se presenta una trombosis superficial, localizada en una de las venas safenas, especiahmente cuando éstas son varicosas, y cuando ha ocurrido una hemorragia ante, intra, o post-partum, de consideración.

A pesar de gue la flebo-trombosis se presenta generalmente de manera precoz, dentro de las dos primeras semanas del paerperio, su evolucion a veces es slenciosa en este periodo inicial de modo que puede pasar inacivertida, y los signos conocidos, como edema dolor fiebre, etc., son inconstantes. Adenas, el trombus cetí mal orgurado es mu 
blando y poec adherente a la pared venosa; por consiguiente las embolias son fáciles. Todos estos caracteres permiten diferenciar la flebotrombosis pura del atro tipo, que vamos a describir a continuación.

$2^{\circ} \mathrm{La}$ verdadera trombo-flebitis, que es el "tipo inflamatorio", caracterizado por los signos habituales de dolor, edema, hipertermia local, y fenómenos febriles, que en general son discretos al comienzo, y precedidos apenas por ligera taquicardia $y$ escalofrios muy moderados. Los signos claros sólo se manifiestan hacia el comienzo de la tercera semana. Es indudable que la trombo-flebitis constituye un proceso infeccioso. Se afirma que en este tipo el coágulo está mejor organiz do, y por consiguiente es más adherente a la pared venosa y menos susceptible de dar embolias.

Asi pues, la distinción entre flebotrombosis $y$ trombo-flebitis se basa cn elementos patogénicos y clínicos. Mientras que la primera es un simple proceso de coagulación, más bien precoz en su aparición, de marcha iscreta $\mathrm{y} \sin$ fenómenos fobriles, 18 segunda se acompaña del elemento éptico, es más tardia. más conspiua, y con fencmenos febriles o subebriles. Sin embargo, este clasificación. teóricamente bastante lógica, resulta en la práctica demasiado esquematica $y$ artificial, y es forzoso dmitir la existencia de casos de interpretacion dificil. Tambien es ob. vo que un proceso iniciads como una imple trombosis, se iransfornara acimente en una tromborlebitis, I sobrevenir la infercon del coisgulo.
El viejo término "phlegmasia alba dolens" debiera tener un lugar muy limitado en la práctica clinica, puesto que los verdaderos casos que corresponden a esa denominación, debidos a obstrucción linfática, son muy raros. Se caracterizan por una linfangitis peri-venosa, originada en los vasos linfaticos cervicales y pélvicos. Sin emibargo, debe tenerse en cuenta que este proceso puede asociarse al de trombosis, constituyendo la vía de propagación séptica que permite la infección del coágulo y el desarrollo de una verdadera trombo. flebitis.

Finalmente debe mencionarse el tipo "trombo-flebitis supurativa" en el cual se produce una constante invasion del torrente sanguíneo por material séptico desprencido del trombus supurado, y se constituye el cuadro de la séptico-piohemia.

Enbolia pulmonar. El peligro de la embolia existe principalmente en el primer dia de la formación del trombus. La profilaxia del accidente consiste en prevenir la formacion de nuevas trombosis, lo cual es mas importante que el tratamiento diri. gido propiamente eontra el proceso ya constituido. Las ienomenos comumente observados son: dolor más o menos agudo en aigun sitio del torax. tos cisprea expectoration sarguinolenta, a veces purulenta, $y$ los signos y sintomas de derrame pleural de absteso del pulmon, o de preumonia la incesidad de estos componentes del sindrome varia mucho. y en codie caso cunlquera de los signes om smos puede hallarse riesustamente cagerado, o disminudo. o aurente wor eoplets. 
Observaciones clinicas. A continuación se presentan algunas notas de un estudio que realicé en el Ham. mersmith Hospital (British Post-Graduate Medical School, University of London), en marzo de 1946, sobre doce casos de embolia pulmonar ocu. rridos en el periodo comprendido entre mayo de 1939 y marzo de 1946, y cuyas historias pertenecen al archivo del Departamento Obstétrico de dicho Hospital.

Los resumenes de estas historias clínicas son los siguientes:

Caso número 1-M. F. 35 años. Gestación a término. Núnero dej embarazo no registrado en la historia. Estenosis mitral. Después de un trabajo de 5 horas 45 minutos, tuvo un parto espontáneo (16 de mayo de 1939). No hubo desgarradura, ni hemorragia. Placenta y membranas completas. En el puerperio tuvo una embolia pulmonar, pero la hi toria no registra pirexia, ni trombosis, ni la fecha del accidente. Salió bien del Hospital.

Caso ninero 2-M. C., 20 años. Frimigestante a término. Trabajo de 53 boras 30 rinutos, que : rminó por un parto espontáneo el 26 de noviembre de 1939 , sin desgarradura. Retención de la placenta, y hemorragia. Extracción manual. Pirexia en al puerperio. En los cultivos de nuestra vaginal se desarrollaron $\mathrm{L}$, Coli y estreptacoco no hemolition. Trombosis en ambas piernas, y embolia pulmonar bilateral (sin registro de fechas). Salió bién.

Caso numero 3-L. B., 32 años. Tercigestante a termino. Un aborto y un parto a término, con niño nacido nuerto. Pelvis androide. Artropetia jelvica. Venas varicosus Rup- tura prematura de las men ranas. Inducción medica $\sin$ resultados. El 11 de julio de 1941, se practico una cesárea segmentaria. No hubo hemorragia, ni pirexia en el puerperio. El 30 de julio de 1941 (curso de la tercera semana), se presento una trombosis superficial en la region anterior de la pierna derecha. El mismo dia sufrio una embolia pulmonar derecha, y el 20 de agosto otra en el pulmón izquierdo. Salió bien el 28 de agosto.

Caso número 4-M. H. 34 años. Primigestante a término. Amenaza de aborto a las 14 semanas. Nefritis crónica. que se manifesto por una hipertensión temprana en el embarazo, azohemia alta, y prueba de "urea clearance" deficiente en el puerperio. Despues de un intento de induccion por ruptura artificial de las membranas, se practico una cesarea segmentaria en las siguientes condiciones: Inercia secundaria, presentación de vertice alta, sufrimiento fetol y albuminuria acentuada. La operación se realizó el 20 de septiembre de 1942, y termino por histerectomía, debido a una hemorrogia incoercible. No hubo pirexia del puerperio E) 22 de septiembre se practicaron cultivos, con los siguientes resultedos: Orina muest tomada con sonda : B. Coli y enterocucos; vagina, Staph albus El 2 de octubre final de la segunda semana), se produjo una cmbolia pulmonar que causó la muerte. En la autopsia se encontró una trombosis de la vena lliaca primitiva aquercia

Cáso numero 5-E. L. 31 ānos. Primgestante, 37 semanas. Hemorragia ante-partum, por placenta baja, variedud marginal Al ingresar, pre- 
intaba contracciones débiles $\mathbf{y}$ presentación cefálica móvil. El 16 de mérzo de 1945, cesárea segmentaria sin complicaciones. No hubo pirexia. al 18 de marzo, cultivos de muestras de nariz y garganta, en los cuales se desarrolló estreptococo hemolítico del grupo A. El 20 de marzo (cuarto dia), signos de trombosis superficial en la parte media del muslo izquierdo (safena). El 28 de marzo (final de la segunda semana), se presentó una embolia pulmonar que causó la muerte.

Caso número 6-M. M. 34 años. Multigestante V, 9 semanas. Sindrome de aborto habitual. Albuminuria. Desde su ingreso, temperatura subfebril en la tarde, ocasionalmente. Todas las investigaciones fueron nesativas. El 24 de noviembre de 1945 , una embolia del pulmón derecho que causó la muerte. La autopsia demostró una trombosis femoral, lado izquierdo.

Caso número 7-L. W. 29 años. Tercigestante, 38 semanas. Estrechez pelvica (pelvis ginecoide pequeña). Frimer embaraze (1942), prueba del trabajo que terminó por cesárea segmenturia Segundo embarazo (1944), cesarea segmentaria electiva, seguira de hemorragia. Ambos puerperios in complicaciones. El 25 de diciemre de 1945 , cesárea segmentaria - lectiva, seguida de esterilización. Murió rueve horas después de la in. tervención. En la autopsia se encontro una embolia gaseosa en la artera pulmonar, aparentemente orif nada en las venas del plejo ovárico quierdo.

Caco número $8-A$ R. 37 años. Primigestante a térnino Dismenorrea artes de casarse. Retmatismo en la infancia. Salpingitis a los 28 años. $\mathrm{Pel}$ V is androide-ginecoide. Trombo-flebitis en la pierna derecha, a la octava semana del embarazo. Toxemia pre-eclámptica. Cesárea segmentaria electiva el 15 de diciembre de 1945. Pirexia iniciada el 6 de enero de 1946 (comienzo de la cuarta semana). Los cultivos de la muestra vaginal revelaron estreptococo no hemolitico, Stanph. albus, $y$ bacilos difteroides. El 5 de enero (final de la tercera semana), signos de trombosis en la pierna derecha, y dos semanas después en la izquierda. La embolía pulmonar lado derecho), s presentó un día antes de los primeros signos de trombosis. La convalecencia fue lenta, pero la enferma salió bien del Hospital.

Caso número 9-S. B., 23 años. Primigestante, 32 semanas. Trabajo de 7 horas 45 minutos, que terminó por parto espontáneo el 29 de diciembre de 1945. No hubo desgerradura. Placenta y membranas comple. ias. No hubo hemorragia. No hubo pirexia. Trombosis el décimo sexto dia, y muerte por embolia pulmonar (no hay fecha registrada).

Caso número $10-A$ - C. 25 anos. Primigestante a termino. Hemorragia ante-partum, y dos exámenes vaginales en la casa, por la partera. Duración del Trabajo. 19 horas. Parto espontineo, episiotomía lado derecho. Placenta y me branas compietas, $\sin$ evidencia de des jendimiento, ni de insercion baja. Hemorragla postpartum de mediana intensidad 10 onzas). Pirexia puerperit, iniciada a las 24 horas; temperatura irregular que se numalizo a ths 42 dias 111 do narzo de 1946 Cultivos de muestas de nuriz y parann- 
ta, negativos, para estreptococo hemolítico. Hemocultivo negativo. El 5 de febrero de 1946 (octavo día), se manifestó una parametritis del lado cerecho. Se quejó por primera vez del muslo derecho y de la pierna izquierda, el 13 de febrero de 1946 (comienzo de la tercera semana), y se desarrollo una trombosis de ambos lados. El 20 de febrero, dolor en la ingle derecha y en el muslo izquierdo. El 27 de febrero (comienzo de la quinta semana), se presentó una embolía en el pulmón derecho; $y$ cos dias después otra en el izquierdo. Salió bien del Hospital.

Caso número 11-E. T., 42 años. Primigestante, 36 semanas. Toxemia pre-eclámptica. Cesárea corporal, el 16 de febrero de 1946. Hemorragia curante el acto operatorio, avaluada en 12 a 15 onzas. No hubo pirexia; los cultivos fueron negativos. El 26 de febrero (segunda semana), signos de trombosis en la pierna izquierda. El 17 de marzo de 1946 qquinta semana), embolía periférica en el pulmón derecho. Salio bien del Hospital.

Caso numero $12-1$. G., 29 años. Primigestante a término. Ruptura prematura de las membranas. Trabajo de 12 horas 50 minutos. Parto espontáneo el 11 de marzo de 1946. Desgarradura perineal de segundo grado. Placenta y membranas completas. Hemorragia post-partum (alrededor de 16 onzas). Pirexia puerperal iniciada el 20 de marzo. En el cultivo de la muestra vaginal se desarrolló $B$. Proteus; en el de la orina (con sonda), B. Coli y Streptococeus foecalis. Trombo is profunda en ambos muslos, aparecida el 19 de márzo tomenzo de la segunda semana).
Dos dias despues, embolia c pulmón derecho. Salió bien.

Los doce casos pueden ser analizados de la siguiente manera:

\section{Palogenia:}

Once casos fueron consecutivos a procesos de trombosis. Uno se debio a embolia gaseosa.

Edad:

Cinco cas por debajo de 30 años. Siete sobre 30 años.

Paridad:

Ocho en primparas.

Tres en multiparas.

Uno no registrado.

Intervenciones:

Seis cesáreas, asi

Un caso de inducción médica infruetuosa, seguida de cesérea segmentaria

Un caso de ruptura artificia! de las membranas, seguida de cesátea segmentaria

Una cesirea segmentaris para hemorragia ante-partum

Dos cesareas segmentaras electivas, une de ellas con esteriliz ción.

Una cesárea corporal electiva.

Cinco partos espontáneos, así:

Uno segu do de extracción manual de la placenta.

Uno procedido por dos eximenes vaginales hechos por una partera, a domicilio.

Tres partos sin intervencion.

Un caso de muerte en ls novena semana del emburazo, sin intervención. 


\section{Prexia:}

Se utilizó como criterio de Pirexia Puerperal el standard del Ministerio de Salud, que rige en las estadisticas de la generalidad de los hospitales ingleses:

"Se clasifica como pirexia puerperal, toda temperatura igual o superior a $38^{\circ} \mathrm{C}$. (100.4 F.), sostenida por 24 horas, o repetida en el curso de las tres semanas que siguen al nacimiento, con excepción de las primeras 24 horas. Las temperaturas deben ser tomadas dos veces por dí (mañana y tarde), por medio de un termómetro sensible al medio minuto, colocado en la boca durante ese minimum de tres minutos".

De acuerdo con este criterio, los casos se distribuyen asi:

Cuatro casos con pirexia.

Cinco sin pirexia.

Un caso no regisirado.

Un caso fue pre-natal, sin fenómenos febriles.

Un caso terminó por muerte nueve horas después de cesárea.

Miero-organismos:

Solamente fueron identificedos en cinco pacientes. Los gérmenes hallados fueron:

B. Coli.

Staphilococcus albus.

Streptococcus tipo no hemolitico. y tipo hemolitico del isupo A).

Enterococcus.

Streptococcus foecalis.

Difteroides.

B. Proteus.

\section{Trombosis:}

Descubierta clínicamente, en ocho casas.

Descubierta post-mortem, en dos casos.

No registrada, en un caso.

Embolia gaseosa, sin trombosis, en un caso.

Embalia pulmonar: (tiempo de aparicion).

Tres casos ocurridos en las dos primeras semanas del puerperio.

Cinco casos ocurridos en la tercera y cuarta semanas.

Una embolia gaseosa nueve horas cespues de cesarea.

Un caso pre-natal.

Si se quiere relacionar entre sí los datos de trombosis, pirexia y embolía, se debe elegir los casos en los cuales la evolución ha sido bien registrada con indicacion de las fechas. Se encuentran siete historias que reúnan esta condición. Ias cuales pueden discriminarse asi:

De los siete casos inumeros 3, 4, $5,8,10,11$ y 12), la trombosis ocurrió precozmente (en el curso de las dos primeras semanas del puenperio) cn cuatro pacientes inumeros 4, 5 , 11 y 12). Fin los tres restantes números 3,3 y 10), la trombosis puede considerarse como tardia $\left(3^{\mathrm{a}}\right.$ y $4^{\mathrm{s}}$ semunas).

En d primer grupo (precoces), tres furon $\sin$ pirexia $(4,5,11)$, y uno (12) con pirexia. En tres de hos la entolia fue precoz (dos primers semenas, numetos 4, 5. 12, y ario fue tardia quinta semará, tomaro 11). 
En el segundo grupo (trombasis tardia), un caso fue sin pirexia (número 3), y dos con pirexia (números 8 y 10). En todos la embolia fue tardia (3* $04^{*}$ semana).

Como el número de observaciones es tan reducido, no puede hacerse intento alguno de utilizar estos datos con el fin de confirmar o negar el valor clínico de la clasificación mencionada al comienzo de este trabajo.

Resultados: Siete casos salieron del Hospital en buenas condiciones. Cinco pacientes murieron a causa de la embolía.

En esta serie se advierte que la embolía ocurrió gerieralmente después de haberse presentado, con mayor o menor intensidad, los sintomas y signos de trombosis. No obstante, éstos pueden faltar, $y$ entonces la complicación pulmonar no es prececida por ningún elemento clínico que haga pensar en su posibilidad (vease el caso número 4). Tal cosa ocurrió también en una paciente de clientela particular, cuya historia se presenta en seguida:

$\mathbf{N}$. N., de 31 años, nacida en Bogotá. Como antecedentes generales de importancia, laparotomia en 1945 , para apendicectomia y correccion de una retro-desviación uterna. Antecedentes ginecológicos: Ciclos norma. les, ligera disnenorrea pre-menstrual, moderada leucorrea. Antecedentes obstétricos: Tres partos espontineos, normales, a término, con niños vivos $y$ puerperios normales. En el último cmbarazo (1947), presentó vómitos cuya intensidad reyuirió reposo en cama y tratamiento con firidoxina e inyeccones intravenusas de dextrosa. Ultima menstrunción, el 8 de marzo de 1949. Du- rante las ocho primeras semanas de este $4^{\circ}$ embarazo, presentó los sintomas siguientes:

\section{Amenorrea.}

Nerviosidad.

Dolores abdominales, casi continuos, que a veces se exacerbaban hasta constituír cólico, con localización especial en la región para-umbilical derecha, $y$ también en las fosas iliacas.

Náuseas, vómitos y constipación.

Subictericia.

Ademas, hubo desde el principio una hipotension (promedio $90 / 60$ ), que se observó durante todo el curso del embarazo, y se trató con "lingüetas" de acetato de desoxicorticosterona.

Desde las 19 semanas se encontró una anemia moderada (Hemoglobina $75 \%$ ). Se trató con sulfato ferroso, por via oral, que no fue tolerado, y se cambio por inyecciones intramusculares de una solución de cacodilato de hierro. También se administró extracto hepatico concentrado crudo, $y$ diversos polivitaminicos.

El 13 de diciembre de 1949, después de un trabajo normal de 8 horas de duración, auvo un parto espontáneo, a termino, con nino vivo, normal, de 3390 gramos. La expulsión de la placenta fue espontanea; placenta y membranas se encontraron completas; peso 570 gramos. No hubo hemorragia. Un pequeño desgarro de la horguilla vulvar fue suturado con dos puntos de catgut simple.

Durante la primera semana del puerperio, transcurrida en la Clinica, la evolución fue completamente a rética. La frocuencia dei rulso 
Le normal, y no hubo signo alguno de importancia, salvo un retardo en la involución uterina, observado hacia el $4^{\circ}$ y $5^{\circ}$ días. Se administró Ersotrate en tabletas durante esos dos dias. Los loquios fueron por completo normales. La lactancia se estableció satisfactoriamente. El $6^{\circ}$ día la p aciente se levantó a una silla, y bandonó la Clínica el $7^{\circ}$ dia, sin cue se hubiese observado sintoma ni signo alguno ue revelase la presencia de trombosis, ni pélvica, ni de los miembros.

La evolución ulterior fue absolutamente normal durante tres dias mis, durante los cuales la paciente caninó moderadamente en su casa. Ei 23 de diciembre, dia undécimo del verperio, se presentó por la mañaun edema en la mejilla izquierda, bido a inflamación de una pieza ntaria. En la tarde del mismo dia areció súbitamente un dolor bastante intenso en la base del hemitórax derecho, que persistió durante toda la noche, acompanado de dispnea, pero sin tos ni expectoración. Pxaminada en la tarde del dia siiente, se comprobó una temperara oral de $38.5^{\circ}$. pulso de $120, y$ -sistencia del dolor en el mismo to especialmente agudo en al dors. base derecha, con dispnea, polip1. a alrededor de 36 respiraciones por minutol, pero $\sin$ tos ni expecación. La tensión articial era norl, y en el examen físico del tórax completamente negativo, 10 misque el del abdonen y los miems. Involución uterina nomal, y uios casi nulos. Se presertió un gesico, $y$ se aplicaron 400.000 dades de penicilina on uns dosis typensión acuosal. Fi sulente dia. después de mejoria poco notable, fue necesario repetir la medicación analgésica y se administró otra dosis de penicilina, 400.000 unidades. La temperatura fue de $37.8^{\circ}$ por la mañana, y se normalizó por la tarde, pero el pulso continuó acelerado, alrededor de 110, por unas dos semanas más. En los dias subsiguientes la evolución fue apirética, $\sin t o s, \sin$ expectoración, sin signos físicos pulmonares, pero el dolor y la dispnea continuaron, si bien en forma muy tolerable. La enferma guardó cama, y el 2 de enero (vigésimo-primero día del puerperio) se autorizó su salida en automóvil para hacer una radiografia del Tórax, que dio el siguiente resultado:

Informe radiológico número 40.849 de la Clinica de Marly:

"En el examen radiológico del tórax se encuentra una leve opacidad de la parte media de la base del pulmón derecho, que tiene alrededor de cuatro por tres y medio centimetros y que se termina en su porción superior por un borde convexo. Me queda la impresión de que esta imagen fue la consecuencia de un pequeño infarto pulmonar de origen embólico".

Firmado, Gonzalo Esguerra Gómez"

Fn vista de este resultado, $y$ a pesar de que el estado de la enferma era completamente satisfactorio, y de que un cxamen muy cuidadoso no revelaba signo alcuno de un proceso de trombosis, se ardenó guardar reposo completo, y una nueva surie de 1200000 undades de pentelina Cono habia una hipogaluctia muy ma* 
nifiesta, se ordenó para el niño una alimentación artificial exclusiva.

El hecho de haberse presentado el 6 de enero (dia vigésimo-quinto) un ligero dolor en la base del hemitórax izquierdo, hizo pensar en una nueva embolia en el otro pulmón. y obligó a prolongar el reposo por una semana más, al cabo de la cual se autori. zó la leventada. Sin embargo, este sintoma fue mucho mas moderado que el primero, y sólo duró unas pocas boras, sin que la enferma presentara en adelante manifestación alguna que entorpeciera su restablecj. miento.

Es este, pues, en resumen, el caso de una multipara que presenta una embolía pulmonar comprobada por los rayos $\mathrm{X}$. en el curso de la segunda semana de un puerperio por 10 demás normal, y en ausencia completa de todo sintoma o signo indicador de un proceso de trombosis. No obstante, éste debió producirse, y la cvolución, en conjunto, hace pensar que el caso en referencia encuadra dentro de la descripción de flebotrombosis. presentada al comienzo de este estudio.

El análisis de este caso trajo a $\mathrm{mi}$ memoria otro parecido, ccurrido anteriormente (febrero de 1948), y al revisar la historia encuentro que se trataba de una multipara $V$, que presentó un cuadro de una notable similitud clínica con el que acaba de ser relatado. El par fue espontaneo, a término, con trabajo de 3 horas de duracion. y una desparradura pecineal de primer grado. Puerperio completamente normel hacta el décimo dia. cuancto se inició un estado febril acompañado de intenso dolor toráxico (base dert is y marcada dispnea, sin tos ni expectoración. En esta enferma se encontraba, por la percusión, una submacicez en la base del pulmón derecho, que se interpretó com una pleuritis. El examen no descubrio ningún signo de trombosis. y el estado febril cesó una semana despuẹs, aunque el dolor, la dispnea ? la taqui cardia se prolongaron por cerca de dos semanas mas. Se trati con penicilina y reposo, y a los cuarenta $y$ dos dias de puerperio se hizo una radiografia que no mostro kormalidad alguna en los campos ilmonares (informe radiológico nutboro 8.771 del doetor Julio Medin Medina). Falta pues el elemento adiológico de certeza con que se co aba en el otro caso, pero la evolución clínica es indudablemente de un parecido extraordinario, y puede aventurarse la opinión de que el segundo descrito corresponde a la misma ntidad, si bien la lesión pulmonar ya no era visible en las radiografias, que fueon hechas mucho mas tarde, 32 dias despues de ocurrida la complicacion, en tanto que en el otro caso se tomaton a los diez lias.

Sumario.

En el presente estudio se mencioniti los elementos clínicos de los procesos de flebo-trombosis, tromboflebitis $y$ embolía pulmonar, en el estado puerperal.

Se presentan las historias clinicas de doce casos de embolia pulmonar ocurridos en el Departamento Obstetrico del Hammersmith Hospital Londres) en un periodo de siete ahos. 
Se analizan estos casos desde los ciguientes puntos de vista:

Patogenia. Edad Paridad. Intervenciones. Pirexia puerperal. Microorganismos. Trombosis. Tiempo de aparición de la embolia. Resultados.

Se relata la historia de una enferma de clientela particular, que presentó una embolía pulmonar el uncécimo día del puerperio, comprobada ulteriormente por los rayos $\mathrm{X}$.

Se relata la historia de otra paciente particular, que presentó un cuaUro clínico interesante por su simili- tud con el anterior, pero en la cual la investigación radiológica dio resuitados negativos.

\section{BIBLIOGRAFIA}

De Lee, Joseph B. Principles and Practice of Obstetrics.

Ramsay, Melvin A and Dolphin, A. The Modern Treatment of Puerperal Sepsis (Medical Press and Circular, number 5586, may 29, 1946. Landon).

Queen Charlotte's Text-Book of Ostetrics, London, 1945.

\title{
PRESENTACION
}

\section{de una técnica original nara las aplicaciones dol Forceps en las variedades posteriores dal vértice}

\author{
Por Rafael Ramirez Merchán \\ Profesor Agregado de Clinica Obstétrica - Facultad de Medicina \\ de la Universiciad Nacional.
}

El mecanismo del parto en las presentaciones posteriores de vértice se resiente de graves inconvenientes por la dificultad de la rotación intrapélvica y del descenso de la presentación; como consecuencia, se prolonga el trabajo con detrimento de la vitalidad fetal $y$ desesperante sufrimiento materno. No obstante, se acostumbra esperar hesta cuando amague el accidente o se presente de hecho. Al paso que las estadicticas estaunidenses anotan que el Forceps se aplica en un $75 \%$ de los casus por "defecto de las furrzas del parto", un porcentaje simiar corres- ponde entre nosotros al "sufrimiento fetal". Esta comparación, nos demuestra que somos demesiado abstencionistas y que es preciso revalidar aspectos rutinarios de nuestras conductas obstétricas. Lo cientifico, to lógico, lo conveniente y lo huma no, es intervenir a tiempo haciendo la profilaxis de las complicaciones $y$ no esperando a que ocuran. Kstos peligros. pródigos en las jresenta. ciones de que venimos hablando, se evitan o tratan de evitarse intervniendo oportuamente. Las manio. bras manuales aconejadas no son precticus en el sentir de la gemertil. 